\title{
Microsymposia
}

MS.60.3

\section{High-resolution X-ray diffraction analysis of strain relaxation in epitaxial oxide thin films}

Rene Guinebretiere ${ }^{1}$, Florine Conchon ${ }^{1}$, Alexandre Boulle ${ }^{1}$, Cecile Girardot $^{2}$, Stephane Pignard ${ }^{2}$, Eric Dooryhee ${ }^{3}$,

Jean Louis Hodeau ${ }^{3}$

${ }^{1}$ Lab. Science des Procedes Ceramique et de Traitements de Surface, ENSCI, 47, Av. A. Thomas, Limoges, Limousin, 87065, France, ${ }^{2}$ LMGP UMR CNRS 5628, INP Grenoble, MINATEC, 3 parvis Louis Néel BP 257, 38016 Grenoble, France, ${ }^{3}$ IN CNRS UPR 2940, 25 avenue des Martyrs BP 166, 38042 Grenoble - France, E-mail : rene.guinebretiere@ unilim.fr

It is now well accepted that epitaxial strains can be used to stabilize out of equilibrium phases [1]. This approach is especially interesting in the case of functional oxide thin films usually subject to many solid-state phase transitions as a function of external constrains (i.e temperature or pressure variations). The precise evaluation of strains and strain gradients is thus one of the main issues in the development of devices based on functional oxides. Highresolution x-ray diffraction (HR-XRD) is one of the most efficient non-destructive methods to extract such information [2]. $\mathrm{RNiO}_{3}$ compounds are extremely difficult to stabilize because of the less stable $3+$ oxidation state of $\mathrm{Ni}$. We have recently shown $[3,4]$ that $\mathrm{SmNiO}_{3}(\mathrm{SNO})$ perovskite can be epitaxially grown on both $\mathrm{SrTiO}_{3}$ and $\mathrm{LaAlO}_{3}$ single crystalline substrates. Those two different cases are corresponding to different lattice mismatch between the film and the substrate. In this communication we present a detailed study of the microstructure of SNO films. This analysis is mainly based on HR-XRD and more specifically on Reciprocal Space Mapping. The contribution of chemical and mechanical effects to the lattice parameters can be rigorously separated using both laboratory and synchrotron HR-XRD. The effect of relaxation-induced misfit dislocations has been investigated and the dislocation densities were derived from the reciprocal space maps. The presence of misfit dislocations induces asymmetrical longitudinal profiles recorded at synchrotron source, those profiles were quantitatively analysed.

[1] N.A.Pertsev et al., PRL, 80 (1998) 1988-1991.

[2] A. Boulle et al., JAC, 36 (2003) 1424-1431

[3] F. Conchon et al., APL, 91 (2007) 192110, 1-3.

[4] F. Conchon et al., JPCM, 20 (2008) 145216, 1-7.

Keywords: strain, high-resolution X-ray diffraction, epitaxial thin films

\section{MS.60.4}

Acta Cryst. (2008). A64, C106

\section{Paramagnetism and ferromagnetism of $\mathrm{TiO}_{2}$ and $\mathrm{ZnO}$ as seen by XMCD: A way to study defects in oxides}

Paolo Imperia $^{1}$, A. Barla ${ }^{2}$, N. H. Hong ${ }^{3}$, J. -P. Kapler ${ }^{4}$, K. R. Whittle ${ }^{1}$, G. R. Lumpkin ${ }^{1}$

${ }^{1}$ ANSTO, Institute of Materials Engineering, New Illawarra Road, Lucas Heights, Sydney, NSW, 2234, ${ }^{2}$ ALBA/CELLS, Barcelona, Spain, ${ }^{3}$ LEMA, Tours, France, ${ }^{4}$ IPCMS, Strasbourg, France, E-mail:plo@ansto.gov.au

$\mathrm{ZnO}$ and $\mathrm{TiO}_{2}$ have attracted in recent years much attention possessing a large range of potential applications. One field is waste form management. Pyrochlores, $\mathrm{A}_{2} \mathrm{Ti}_{2} \mathrm{O}_{7}$ fluorite based materials have been considered for long-term stabilization of high and medium level nuclear waste, including future generation-IV nuclear technologies. Understanding the basic physics of radiation damage is undeniable and the quest should clearly start from simple systems like
$\mathrm{TiO}_{2}$ and $\mathrm{ZnO}$. The magnetic properties in both compounds can be related to the presence of oxygen vacancies. X-ray magnetic circular dichroism (XMCD) gives a direct proof of it conjugating the atomic specificity and sensitivity of a synchrotron based spectroscopic technique to the magnetic properties. $\mathrm{TiO}_{2}$ is a wide gap diamagnetic semiconductor. If an oxygen vacancy is created, to compensate the charge a $\mathrm{Ti}^{4+}$ cation must change from the diamagnetic $\mathrm{Ti}^{4+}$ to a paramagnetic $\mathrm{Ti}^{2+}$ or two $\mathrm{Ti}^{4+}$ cations must change to $\mathrm{Ti}^{3+}$. We studied the magnetic properties of doped and undoped $\mathrm{TiO}_{2}$ and $\mathrm{ZnO}$ measuring the dichroic signal at the $\mathrm{Ti} \mathrm{L}_{2,3}$ edges and $\mathrm{O}$ and $\mathrm{Zn} \mathrm{K}$ edges. Evidence of ferromagnetism at the $\mathrm{K}$ edge of $\mathrm{O}$ and at the $\mathrm{L}_{2,3}$ edges of Ti associated with oxygen vacancies, has been found, while no ferromagnetic behavior has been observed at the $\mathrm{K}$ edge of $\mathrm{Zr}$. From the magnetic properties information could be obtained about the defects. A further step is the study of irradiated samples with opportunely induced defects. This will highlight trough the evolution of the magnetic properties the irradiation induced defect formation. Here we set forth a conceptually different approach; instead to evaluate the crystalline to amorphous fraction we propose to analyze magnetic defects created by irradiation in otherwise diamagnetic crystals.

Keywords: thin-film properties, defects, magnetic properties

\section{MS.60.5}

Acta Cryst. (2008). A64, C106-107

\section{Mn atoms in GaAs: First evidence for Ga interstitial site occupation}

Krystyna Lawniczak-Jablonska ${ }^{1}$, Anna Wolska ${ }^{1}$, Marcin T Klepka ${ }^{1}$, Janusz Sadowski ${ }^{1,2}$, Elisabeth Holub-Krappe ${ }^{3}$, Andreas Persson ${ }^{4}$, Dimitri Arvanitis ${ }^{4}$

${ }^{1}$ Polish Academy of Sciences, Institute of Physics, Al. Lotnikow 32/46, Warsaw, Poland, 02 668, Poland, ${ }^{2}$ Lund University, Max-Lab, Lund SE-221 00, Sweden, ${ }^{3} \mathrm{Hahn}-$ Meitner Institute, Department of Magnetism, Glienicker Str. 100, D-14109 Berlin, Germany, ${ }^{4}$ Physics Department, Uppsala University, Box 530, 75121 Uppsala, Sweden, E-mail : jablo@ ifpan.edu.pl

$\mathrm{Ga}_{1-\mathrm{x}} \mathrm{Mn}_{\mathrm{x}} \mathrm{As}$ is commonly considered as a promising material for microelectronic applications utilizing the electron spin. The location of the $\mathrm{Mn}$ atoms in the by MBE grown layers is correlated with all important physical properties of the final material, therefore it is the subject of many studies. A powerful tool for this kind of study is $\mathrm{x}$-ray absorption spectroscopy (XAS) as it probes the local atomic order and the electronic structure. We calculate the influence of the $\mathrm{Mn}$ atom location within the GaAs matrix on the shape of the XANES spectra by means of the ab initio calculations using FEFF 8.4. Different Mn positions within the GaAs matrix were considered: (a) substitutional MnGa, (b) interstitial (As); with As atoms as the first neighbours, (c) interstitial $(\mathrm{Ga})$; with $\mathrm{Ga}$ atoms as the first neighbours. The theoretical predictions were compared with the experimental $\mathrm{K}$ and L edge XANES of Mn measured on the samples without any thermal treatment and after annealing to different temperatures. It is shown that in the considered samples the Mn atoms may occupy more than one position in the crystal lattice, therefore a superposition of possible locations was considered. This allows to determine the possible distribution of $\mathrm{Mn}$ between all possible lattice locations. We find for the first time that $\mathrm{Mn}$ atoms clearly prefer the Ga interstitial positions.

Acknowledgments

This work is partially supported by the Polish National Grant of the Ministry of Science and High Education N202-052-32/1189 as well as by DESY/HASYLAB, MAX-lab (EC support program: Transnational Access to Research Infrastructures) and directly by the 